\author{
IMAGE \\ UNAVAILABLE \\ FOR COPYRIGHT \\ REASONS
}

\title{
Serratia's edge
}

Pictured above is the structure of the endonuclease from the enterobacterium Serratia marcescens (see Krause et al. p.p. 461-468, this issue). Serratia infections are becoming an increasingly common side-effect of visits to hospital, causing pneumonia and urinary tract infections. These bacteria secrete the red pigment prodigiosin which can look distressingly like blood, hence the colloquial name for one such disease - 'red diaper syndrome'. A significant contribution to Serratia's pathogenicity is made by a number of 'virulence factors', extracellular enzymes of which this endonuclease is one.

A characteristic of the endonuclease is its broad substrate specificity. The enzyme can digest both single and double stranded RNA and DNA without any apparent sequence preference. Serratia endonuclease has no significant sequence homology with the other broad specificity endonuclease from Staphylococcus, or with any other nuclease whose structure has been determined. Indeed, the threedimensional structure of the Serratia endonuclease reveals that it possesses a unique protein fold.

Although the structure of the endonuclease complexed with nucleic acid has not been determined, consideration of the electrostatic surface of the enzyme has led to the proposal of a model for its nucleic-acid binding site and active site. There is a strongly polarized cleft between a long $\alpha$-helix and loop region (on the right in the picture) which modelling shows can easily accommodate doublestranded DNA. Comparison of the sequences of a number of similar nucleases (whose 3D structures are as yet unknown) has implicated a histidine residue in the active site, and this histidine is located in the putative binding cleft. Determining the basis of the enzyme's broad specificity, and definitive identification of the active site region of the protein, will of course have to await the elucidation of the structure of the nuclease-nucleic acid complex.

[This figure was produced by Tod D. Romo of Rice University and Mitchell D. Miller of the University of Houston. Graphics primitives from RIBBONS (M. Carson) were rendered using RenderMan v3.4 (Pixar) on a Silicon Graphics Indigo (R4000). This rendering system was also used to produce special effects for the motion picture 'The Abyss'.] 